\title{
Phase separation in systems with charge ordering
}

\author{
M.Yu. Kagan ${ }^{(a)},(b)$, K.I. Kugel ${ }^{(c)}$ and D.I. Khomskii ${ }^{(d)}$ \\ (a) P.L. Kapitza Institute for Physical Problems, Russian Academy of Sciences, Kosygina Str. 2, 117334 Moscow, Russia \\ (b) Max-Planck-Institut für Physik Komplexer Systeme, Nöthnitzer Str. 38, D-01187 Dresden, Germany \\ (c) Institute of Theoretical and Applied Electrodynamics, Russian Academy of Sciences, Izhorskaya Str. 13/19, \\ 127412 Moscow, Russia \\ (d) Laboratory of Applied and Solid State Physics, Materials Science Center, University of Groningen, Nijenborgh 4, \\ 9747AG Groningen, The Netherlands
}

\begin{abstract}
A simple model of charge ordering is considered. It is shown explicitly that at any deviation from half-filling $(n \neq 1 / 2)$ the system is unstable with respect to phase separation into charge ordered regions with $n=1 / 2$ and metallic regions with smaller electron or hole density. Possible structure of this phase-separated state (metallic droplets in a charge-ordered matrix) is discussed. The model is extended to account for the strong Hund-rule onsite coupling and the weaker intersite antiferromagnetic exchange. An analysis of this extended model allows us to determine the magnetic structure of the phase-separated state and to reveal the characteristic features of manganites and other substances with charge ordering.

PACS numbers: 71.45.Lr, 75.10.-b, 75.30.Mb, 75.30.Kz
\end{abstract}

\section{INTRODUCTION}

The problem of charge ordering $(\mathrm{CO})$ in magnetic oxides attracts an attention of theorists since the discovery of Verwey transition in magnetite in the end of thirties [1]. An early theoretical description of this phenomenon was given e.g. in [2]. Recently this problem was reexamined in a number of papers in connection with the colossal magnetoresistance in manganites, see e.g. [3 5]. The mechanisms stabilizing the $\mathrm{CO}$ state may be different: the Coulomb repulsion of charge carriers (the energy minimization requires keeping the carriers as far away as possible, similar to the Wigner crystallization), or the electron-lattice interaction leading to the effective repulsion of electrons at the nearest neighbor sites. In all cases, charge ordering can arise in systems with the mixed valence if the electron bandwidth is sufficiently small - large electron kinetic energy stabilizes the homogeneous metallic state. In real materials, in contrast to the Wigner crystallization, the underlying lattice periodicity determines the preferential types of $\mathrm{CO}$. Thus, in the simplest bipartite lattice, to which belong the colossal magnetoresistance manganites of the type $\mathrm{R}_{1-x} \mathrm{~A}_{x} \mathrm{MnO}_{3}\left(\mathrm{R}=\mathrm{La}, \mathrm{Pr} ; \mathrm{A}=\mathrm{Ca}\right.$, Sr), or layered manganites $\mathrm{R}_{2-x} \mathrm{~A}_{x} \mathrm{MnO}_{4}, \mathrm{R}_{2-2 x} \mathrm{~A}_{1+2 x} \mathrm{Mn}_{2} \mathrm{O}_{7}$, the optimum conditions for the formation of the CO state exist for doping $x=1 / 2$. At such value of $x$ the concentrations of $\mathrm{Mn}^{3+}$ and $\mathrm{Mn}^{4+}$ are equal, and the simple checkerboard arrangement is possible. The most remarkable experimental fact here is that even at $x \neq 1 / 2$ (in the underdoped manganites, $x<1 / 2$ ) only the simplest version of charge ordering is experimentally observed with alternating checkerboard structure of occupied and empty sites in the basal plane [6]. In other words, this structure corresponds to the doubling of the unit cell, whereas the more complicated structures with longer period (or even incommensurate structures) do not actually appear in this case.

Then, the natural question arises: how could we redistribute the extra or missing electrons in the case of arbitrary doping level, keeping the superstructure the same as for $x=1 / 2$ ? To answer this question, the experimentalists introduced the concept of incipient charge ordered state corresponding to the distortion of long-range CO by microscopic metallic clusters [7]. In fact, the existence of such a state implies a kind of phase separation. Note that the phase separation scenario in manganites is very popular now [8]- [15]. Nowadays, there is a growing evidence suggesting that an interplay between the charge ordering and the tendency toward phase separation plays an essential role in the physics of materials with colossal magnetoresistance.

In this paper, we consider a simple model, which allows us to clarify the situation at arbitrary doping. We include in this model both the Coulomb repulsion of electrons on the neighboring sites and the magnetic interactions responsible for the magnetic ordering of manganites. After demonstrating the instability of the system toward phase separation in certain ranges of doping, we also consider the simplest form of the phase separation - the formation of metallic droplets in an insulating matrix, estimate parameters of such droplets, and construct the phase diagram illustrating the interplay between charge ordering, magnetic ordering, and phase separation. 
One has to note that the mechanism of the charge ordering considered below (the Coulomb repulsion) is not the only one. The electron-lattice interaction can also play an important role, see e.g. [16]. In application to manganites, one has to take into account also orbital and magnetic interactions [4, 16, 17]. These may be important, in particular, to explain the fact that the charge ordering in half-doped perovskite manganites is a checkerboard one only in the basal plane, but it is "in-phase" in the $c$ - direction. However, the nature of such charge ordering is not clear yet and presents a separate problem: it is not evident that the magnetic interactions responsible for this stacking of $a b$-planes in [16] is indeed the dominant mechanism. Let us also emphasize that the charge ordering in manganites is often observed at higher temperatures than the magnetic ordering, and one has to look for a model not relying heavily on magnetic interactions. Note that in contrast to magnetic interactions, the Coulomb interaction is one of the important factors always present in the systems under consideration. Moreover, it has a universal nature and does not depend critically on specific features of a particular system. Consequently, our treatment can be applied also to other systems with the charge ordering such as magnetite $\mathrm{Fe}_{3} \mathrm{O}_{4}[1]$, cobaltites [18], nickelates [19], etc.

\section{THE SIMPLEST MODEL FOR CHARGE ORDERING}

Let us consider a simple lattice model for charge ordering:

$$
\hat{H}=-t \sum_{<i, j>} c_{i}^{+} c_{j}+V \sum_{<i, j>} n_{i} n_{j}-\mu \sum_{i} n_{i}
$$

where $t$ is the hopping integral, $V$ is the nearest neighbor Coulomb interaction (similar $n n$ repulsion can be also obtained via the interaction with the breathing-type optical phonons); $\mu$ is the chemical potential, and $c_{i}^{+}$and $c_{j}$ are one-electron creation and annihilation operators, $n_{i}=c_{i}^{+} c_{i}$. Symbol $<i, j>$ denotes the summation over the nearest-neighbor sites. Here, we omit for simplicity spin indices. We also assume the absence of a double occupancy in this model due to the strong onsite repulsion between electrons.

The models of the type (1) with the $n n$ repulsion being responsible for the charge ordering are the most popular ones to describe this phenomenon, see e.g. [2,3,5,20] and references therein. Hamiltonian (1) captures the main physical effects; if necessary, one can add to it some extra terms, which we will also do in Section V below.

In the main part of our paper, we will always speak about electrons. However in application to real manganites we will mostly have in mind less than half-doped (underdoped) systems of the type $\mathrm{R}_{1-x} \mathrm{~A}_{x} \mathrm{MnO}_{3}$ with $x<1 / 2$. Thus, for a real system one has to substitute holes for our electrons. All the theoretical treatment definitely remains the same (from the very beginning we could define operators $c$ and $c^{+}$in (1) as the operators of holes); we hope that it will not lead to any misunderstanding.

We consider below the simplest case of square (2D) or cubic (3D) lattices, where for $x=1 / 2$ the simple twosublattice ordering would take place. As mentioned in the Introduction, this is the case in layered manganites, whereas the ordering in 3D perovskite manganites is like this only in the basal plane, the ordering being "in-phase" in the $c$ direction. To account for this behavior, apparently a more complicated model would be necessary.

For the case $n=1 / 2$, the model (1) was analyzed in many papers; we follow the treatment of the Ref. [2]. As mentioned above, the Coulomb repulsion (second term in (1)) stabilizes charge ordering in the form of checkerboard arrangement of occupied and empty sites, whereas the first term (band energy) opposes this tendency. At arbitrary values of electron density $n$, we shall at first consider a homogeneous CO solution and use the same ansatz as in [2], namely

$$
n_{i}=n\left[1+(-1)^{i} \tau\right]
$$

Such an expression implies the doubling of lattice periodicity, with the local densities $n_{1}=n(1+\tau)$ and $n_{2}=n(1-\tau)$ at the neighboring sites. Note that at $n=1 / 2$ for a general form of electron dispersion without nesting, the CO state exists only at sufficiently strong repulsion $V>2 t$ [2]. The order parameter is $\tau<1$ for finite $V / 2 t$, and the ordering in general is not complete, i.e. an average electron density $n_{i}$ differs from zero or one even at $T=0$.

We use the same coupled Green function approach as in [2], which yields

$$
\left\{\begin{array}{c}
(E+\mu) G_{1}-t_{k} G_{2}-z V n(1-\tau) G_{1}=\frac{1}{2 \pi} \\
(E+\mu) G_{2}-t_{k} G_{1}-z V n(1+\tau) G_{2}=0
\end{array}\right.
$$


where $G_{1}$ and $G_{2}$ are the Fourier transforms of the normal lattice Green functions $G_{i l}=\left\langle\left\langle c_{i} c_{l}^{+}\right\rangle\right\rangle$for sites $i$ and $l$ belonging respectively to one or different sublattices, $z$ is the number of nearest neighbors and $t_{k}$ is the Fourier transform of a hopping matrix element. While deriving (3), we performed a mean-field decoupling and replaced the averages $\left\langle c_{i}^{+} c_{i}\right\rangle$ by the onsite densities $n_{i}=n\left[1+(-1)^{i} \tau\right]$. Solution of (3) leads to the following spectrum:

$$
E+\mu=V n z \pm \sqrt{(V n \tau z)^{2}+t_{k}^{2}}=V n z \pm \omega_{k} .
$$

The spectrum defined by (4) resembles the spectrum of superconductor and hence the first term under the square root is analogous to the superconducting gap squared. In other words, we can introduce the charge-ordering gap by the formula

$$
\Delta=V n \tau z
$$

It depends upon density not only explicitly, but also via the density dependence of $\tau$.

Hence, we get

$$
\omega_{k}=\sqrt{\Delta^{2}+t_{k}^{2}}
$$

Note that there is one substantial difference between the spectrum of charge ordered state (5) and superconducting state, namely here for $n \neq 1 / 2$ the chemical potential does not enter under the square root in (5) in contrast to the spectrum of superconductor where

$$
\omega_{k}=\sqrt{\left(t_{k}-\mu\right)^{2}+\Delta^{2}}
$$

Then, we can find the following expressions for the Green functions :

$$
\left\{\begin{array}{c}
G_{1}=\frac{A_{k}}{E+\mu-V n z-\omega_{k}+i 0}+\frac{B_{k}}{E+\mu-V n z+\omega_{k}+i 0} \\
G_{2}=\frac{t_{k}}{2 \omega_{k}} \frac{1}{2 \pi}\left[\frac{1}{E+\mu-V n z-\omega_{k}+i 0}-\frac{1}{E+\mu-V n z+\omega_{k}+i 0}\right],
\end{array}\right.
$$

where

$$
A_{k}=\frac{1}{4 \pi}\left(1-\frac{\Delta}{\omega_{k}}\right), \quad B_{k}=\frac{1}{4 \pi}\left(1+\frac{\Delta}{\omega_{k}}\right) .
$$

After the standard Wick transformation $E+i 0 \rightarrow i E$ in the expression for $G_{1}$, we can find the densities in the following form

$$
\begin{aligned}
& n_{1}=n(1+\tau)=\int\left[\left(1-\frac{\Delta}{\omega_{k}}\right) f_{F}\left(\omega_{k}-\mu+V n z\right)+\left(1+\frac{\Delta}{\omega_{k}}\right) f_{F}\left(-\omega_{k}-\mu+V n z\right)\right] \frac{d^{3} \mathbf{k}}{2 \Omega_{B Z}} \\
& n_{2}=n(1-\tau)=\int\left[\left(1+\frac{\Delta}{\omega_{k}}\right) f_{F}\left(\omega_{k}-\mu+V n z\right)+\left(1-\frac{\Delta}{\omega_{k}}\right) f_{F}\left(-\omega_{k}-\mu+V n z\right)\right] \frac{d^{3} \mathbf{k}}{2 \Omega_{B Z}},
\end{aligned}
$$

where $f_{F}(y)=1 /\left(e^{y / T}+1\right)$ is the Fermi distribution function, and $\Omega_{B Z}$ is the volume of the first Brillouin zone.

Summing up and subtracting two equations for $n_{1}$ and $n_{2}$, we get the resulting system of equations for $n$ and $\mu$ :

$$
\left\{\begin{array}{c}
1=V z \int \frac{1}{\omega_{k}}\left[f_{F}\left(-\omega_{k}-\mu+V n z\right)-f_{F}\left(\omega_{k}-\mu+V n z\right)\right] \frac{d^{3} \mathbf{k}}{2 \Omega_{B Z}} \\
n=\int\left[f_{F}\left(-\omega_{k}-\mu+V n z\right)+f_{F}\left(\omega_{k}-\mu+V n z\right)\right] \frac{d^{3} \mathbf{k}}{2 \Omega_{B Z}} .
\end{array}\right.
$$

For low temperatures $T \rightarrow 0$ and $n \leq \frac{1}{2}$ it is reasonable to assume that $\mu-V n z$ is negative. Hence $f_{F}\left(\omega_{k}-\mu+V n z\right)=$ 0 and $f_{F}\left(-\omega_{k}-\mu+V n z\right)=\theta\left(-\omega_{k}-\mu+V n z\right)$ is the step function.

It is easy to see that for $n=\frac{1}{2}$ the system of equations (9) yields identical results for all $-\Delta \leq \mu-V n z \leq \Delta$. From this point of view, $n=1 / 2$ is a point of indifferent equilibrium. For infinitely small deviations from $n=1 / 2$, that is, for densities $n=1 / 2-0$, the chemical potential should be defined as $\mu=-\Delta+V z / 2=V z / 2 \cdot(1-\tau)$. If we consider 
a strong coupling case $V \gg 2 t$ and assume a constant density of states inside the band, then for a simple cubic lattice we have $\tau=1-\frac{2 W^{2}}{3 V^{2} z^{2}}$, and hence

$$
\mu=\frac{W^{2}}{3 V z}
$$

where $W=2 z t$ is the bandwidth. Note that for the density $n=1 / 2$ a charge-ordering gap $\Delta$ appears for an arbitrary interaction strength $V$. This is due to the existence of nesting in our simple model. In the weak coupling case $V \ll 2 t$ and with the perfect nesting, we have $\Delta \sim W \exp \left\{-\frac{W}{V z}\right\}$ and $\tau$ is exponentially small. For $z V \gg W$ or accordingly for $V \gg 2 t: \Delta \approx V z / 2$ and $\tau \rightarrow 1$. As mentioned above, for a general form of electron dispersion without nesting the charge ordering exists only if the interaction strength $V$ exceeds certain critical value of the order of bandwidth $W$ [2]. Further on, we restrict ourselves only to the physically more instructive case of strong coupling $V \gg 2 t$.

Now let us consider the case $n=1 / 2-\delta$, where $\delta \ll 1$ is a deviation from density $n=1 / 2$. For this case $\mu=\mu(\delta, \tau)$, and we have two coupled equations for $\mu$ and $\tau$. As a result,

$$
\mu(\delta) \approx V n z(1-\tau)-\frac{4 W^{2}}{V z} \delta^{2} \approx \frac{W^{2}}{3 V z}+\frac{4 W^{2}}{3 V z} \delta+O\left(\delta^{2}\right)
$$

Correspondingly, the energy of a charge ordered state is as follows

$$
E_{C O}(\delta)=E_{C O}(0)-\frac{W^{2}}{3 V z} \delta-\frac{2 W^{2}}{3 V z} \delta^{2}+O\left(\delta^{3}\right),
$$

where $E_{C O}(0)=-\frac{W^{2}}{6 V z}$ is the energy precisely for density $n=1 / 2$ and $\left|E_{C O}(0)\right| \ll W$. At the same time, the charge-ordering gap $\Delta$ is given by:

$$
\Delta \approx \frac{V z}{2}\left[1-2 \delta-\frac{2 W^{2}}{3 V^{2} z^{2}}(1+4 \delta)\right]
$$

Actually, the dependence of the chemical potential $\mu$ and the total energy $E$ on $\delta$, Eqs. (11), (12), stems from this linear decrease of energy gap $\Delta$ with deviation from half-filling.

For $n>1 / 2$ the energy of charge ordered state starts to increase rapidly due to the large contribution from Coulomb repulsion (the upper Verwey band is partially filled for $n>1 / 2$ ). Contrary to the case $n=1 / 2$, for $n>1 / 2$ each extra electron put into the checkerboard CO state necessarily has occupied nearest neighbor sites, increasing the total energy on $V z|\delta|$. As a result, we have for $|\delta|=n-1 / 2>0$

$$
E_{C O}(\delta)=E_{C O}(0)+\left(V z-\frac{W^{2}}{3 V z}\right)|\delta|-\frac{2 W^{2}}{3 V z} \delta^{2}+O\left(\delta^{3}\right) .
$$

Accordingly, the chemical potential has the form

$$
\mu(\delta)=V z-\frac{W^{2}}{3 V z}-\frac{4 W^{2}}{3 V z}|\delta|+o\left(\delta^{2}\right) .
$$

It undergoes a jump equal to $V z$ for $\tau \rightarrow 1$. Note that the gap $\Delta$ is symmetric for $n>1 / 2$ and is given by

$$
\Delta \approx \frac{V z}{2}\left[1-2|\delta|-\frac{2 W^{2}}{3 V^{2} z^{2}}(1+4|\delta|)\right] .
$$

We could make the whole picture symmetric with respect to $n=1 / 2$ by shifting all the one-electron energy levels and the chemical potential by $V z / 2$, i.e., defining $\mu^{\prime}=\mu-V z / 2$. This change of variables, of course, would not modify our conclusions.

\section{PHASE SEPARATION}

The most remarkable implication of (11)-(15) is that the compressibility $\kappa$ of the homogeneous charge ordered system is negative for densities different from $1 / 2$, 


$$
\frac{1}{\kappa} \propto \frac{d \mu}{d n}=-\frac{d \mu}{d \delta}=\frac{d^{2} E}{d \delta^{2}}=-\frac{4 W^{2}}{3 V z}<0
$$

where $\delta=1 / 2-n$. This is the manifestation of the tendency toward phase separation characteristic of the charge ordered system with $\delta \neq 0$. The presence of a kink in the $E_{C O}(\delta)$ (cf. Eqs. (12), (14)) implies that one of the states, into which the system might separate, would correspond to the checkerboard CO state with $n=1 / 2$, whereas the other would have a certain density $n^{\prime}$ smaller or larger than $1 / 2$. This conclusion resembles that of [4] (see also [10,14), although the detailed physical mechanism is different. The possibility of phase separation in the model (1) away from half-filling was also reported earlier in 12] for the infinite-dimensional case. Below we concentrate our attention on the situation with $n<1 / 2$ (underdoped manganites); the case $n>1 / 2$ apparently has certain special properties - the existence of stripe phases etc. [13], the detailed origin of which is not yet clear.

It easy to understand the physics of phase separation in our case. As follows from (13), the CO gap decreases linearly with the deviation from the half-filling. Correspondingly, the energy of the homogeneous CO state rapidly increases, and it is more favorable to "extract" extra holes from the CO state, putting them into one part of the sample, while creating the "pure" checkerboard CO state in the other part of it. The energy loss due to such redistribution of holes is overcompensated by the gain provided by the better charge ordering.

However, the hole-rich regions would not be completely "empty," like pores (clusters of vacancies) in crystals: we can gain an extra energy by "dissolving" in them a certain amount of electrons. By doing this we decrease the band energy of the electrons due to their delocalization. Thus, this second phase would be a metallic one. The simplest state of this kind is a homogeneous metal with the electron concentration $n_{m}$. This concentration, as well as the relative volume of the metallic and $\mathrm{CO}$ phases, can be easily calculated by minimizing the total energy of the system. The energy of the metallic part of the sample $E_{m}$ is given by

$$
E_{m}=-t z n_{m}+c t\left(n_{m}\right)^{5 / 3}+V\left(n_{m}\right)^{2}
$$

where $c$ is some constant.

Minimizing (17) with respect to $n_{m}$, we find the equilibrium electron density in the metallic phase. For the strong coupling $V>z t$, we get

$$
n_{m 0}=t z / 2 \mathrm{~V}
$$

Thus, in this simple treatment, the system with $n_{m 0}<n<1 / 2$ would undergo phase separation into the CO phase with $n=1 / 2$ and the metallic phase with $n=n_{m 0}$. Relative volumes $v_{m}$ and $v_{C O}$ of these phases for arbitrary $n$ can be found from the Maxwell construction:

$$
v_{m} / v_{C O}=(1 / 2-n) /\left(n-n_{m 0}\right),
$$

from which we find that the metallic phase occupies the part $v_{m}$ of the total volume $v$ given by the relationship

$$
v_{m} / v=(1 / 2-n) /\left(1 / 2-n_{m 0}\right),
$$

The metallic phase would occupy the whole sample when the total electron density $n$ is less than $n_{m 0}$.

\section{AN EXAMPLE: THE PHASE SEPARATED STATE WITH METALLIC DROPLETS}

As we argued above, the system with the short-range repulsion (1) is unstable toward phase separation for $n$ close but different from $1 / 2$. The long-range Coulomb forces would however prevent the full phase separation into large regions containing all extra holes and the pure $n=1 / 2$ charge ordered region. We can avoid this energy loss by forming, instead of one big metallic phase with many electrons, the finite metallic clusters with smaller number of them. The limiting case would be a set of spherical droplets, each containing one electron. This state is similar to magnetic polarons ("ferrons") considered in the problem of phase separation in doped magnetic insulators [8, 14, 11].

We present below the estimate for the characteristic parameters of these droplets. The main aim of this treatment is to demonstrate that the state constructed in such a way will have the energy lower than the energy of the homogeneous state, even if we treat these droplets rather crudely and do not optimize all their properties. In particular, we will make the simplest assumption that the droplets have sharp boundaries and that the charge ordered state outside these droplets is not modified in their vicinity. This state can be treated as a variational one: if we optimize the structure of the droplet boundary, its energy would only decrease. 
The energy (per unit volume) of the droplet state with the concentration $n_{d}$ of droplets can be written in total analogy with the ferron energy in the double exchange model (see [14]11]). This yields

$$
E_{\text {droplet }}=-t n_{d}\left(z-\frac{\pi^{2} a^{2}}{R^{2}}\right)-\frac{W^{2}}{6 V z}\left[1-n_{d} \frac{4}{3} \pi\left(\frac{R}{a}\right)^{3}\right] .
$$

Here, $a$ is the lattice constant and $R$ is the droplet radius. The first term in (21) corresponds to the gain in kinetic energy of electron delocalization inside the metallic droplets, and the second term describes the charge ordering energy in the remaining insulating part of the sample.

Actually, one should include the surface energy contribution to the total energy of the droplet. The surface energy should be of the order of $W^{2} R^{2} / V$. For large droplets, this contribution is small compared to the term $\propto R^{3}$ in $(21)$; it would also be reduced for a "soft" droplet boundary. One can show that even in the worst case of a small droplet (of the order of a few lattice constants) with the sharp boundary, this contribution would not exceed about 20 percent of the bulk contribution. That is why we will ignore this term below.

Minimization of the energy in (21) with respect to $R$ gives

$$
\frac{R}{a} \propto\left(\frac{V}{t}\right)^{1 / 5}
$$

The critical concentration $n_{d c}$ corresponds to the configuration where metallic droplets start to overlap, i.e. where the volume of the CO phase ( the second term in (21)) tends to zero. Hence,

$$
n_{d c}=\frac{3}{4 \pi}\left(\frac{a}{R}\right)^{3} \propto\left(\frac{t}{V}\right)^{3 / 5} .
$$

By comparing (12) with (21), (22), we see that for the deviations from the half-filling $0<\delta \leq \delta_{c}=1 / 2-n_{d c}$ the energy of the phase separated state is always lower than the energy of the homogeneous charge ordered state. The energy of the droplet state (21) with the radius given by (22) is also lower than the energy of the fully phase separated state, obtained by the Maxwell construction from the homogeneous metallic state (17). Correspondingly, the critical concentration $n_{d c}(23)$ is larger than $n_{m 0}(18)$. There is no contradiction here: the droplet state, which we constructed has electrons confined in spheres of radius $R$, and even when these droplets start to overlap at $n=n_{d c}$, occupying the whole sample, the electrons in this state, by construction, are still confined within their own spheres and avoid each other. In other words, in our droplet state certain degree of charge-ordering correlations is still present, decreasing the repulsion and hence the total energy.

Thus, the energy of a phase separated state with the droplets corresponds to the global minima of the energy for all $0<\delta \leq \delta_{c}$. This justifies our conclusion about phase separation into charge ordered state with $n=1 / 2$ and a metallic state with small spherical droplets.

The situation here resembles that of partially filled strongly interacting Hubbard model, with the CO state corresponding to an antiferromagnetic state of the latter and with the nearest-neighbor interaction $V$ playing the role of the Hubbard's $U$. In both cases, the kinetic energy of doped carriers tends to destroy this "antiferro" or charge ordering, first "spoiling" it in their vicinity and finally leading to the formation of the metallic state (Nagaoka ferromagnetism). In the Hubbard model, we also face the situation with phase separation at a small enough doping [21].

Note also that for $n>1 / 2$ the compressibility of the charge- ordered state is again negative $\frac{1}{\kappa}=\frac{d^{2} E}{d \delta^{2}}=-\frac{4 W^{2}}{3 V z}<0$ and has the same value as for the case $n<1 / 2$. As a result, it is more favorable again to create a phase-separated state for these densities. However, as it was already mentioned, the nature of the second phase with $n>1 / 2$ is not quite clear at present, and therefore we do not consider this case here.

\section{AN EXTENDED MODEL}

Now we can extend the model discussed in the previous sections by taking into account the essential magnetic interactions. In manganites, besides the conduction electrons in $e_{g}$ bands, there exist also practically localized $t_{2 g}$ electrons, which we now include to our consideration. The corresponding Hamiltonian has the form

$$
\hat{H}=-t \sum_{<i, j>, \sigma} c_{i \sigma}^{+} c_{j \sigma}+V \sum_{<i, j>} n_{i} n_{j}-J_{H} \sum_{i} \mathbf{S}_{i} \sigma_{i}+J \sum_{<i, j>} \mathbf{S}_{i} \mathbf{S}_{j}-\mu \sum_{i} n_{i},
$$


In comparison to (1), the additional terms here correspond to the strong Hund-rule onsite coupling $J_{H}$ between localized spins $\mathbf{S}$ and the spins of conduction electrons $\sigma$, and a relatively weak Heisenberg antiferromagnetic (AFM) exchange $J$ between neighboring local spins. In real manganites, the AFM ordering of the CE type in the CO phase is determined not only by the exchange of localized $t_{2 g}$ electrons but to a large extent by the charge- and orbitallyordered $e_{g}$ electrons themselves. For simplicity, we ignore this factor here and assume the superexchange interaction to be the same both in the $\mathrm{CO}$ and in the metallic phases.

It is physically reasonable to consider this model in the limit

$$
J_{H} S>V>W>J S^{2} .
$$

In the absence of the Coulomb term, this is exactly the conventional double exchange model (see e.g. [8,14]). Note that the absence of doubly occupied sites in (20) is guaranteed by the large Hund's term. It also favors the metallicity in the system, since the effective bandwidth in our problem depends upon the magnetic order. Therefore, the estimate for the critical concentration changes here in comparison to (23). Similar to 14 the metallic droplets will be ferromagnetic (FM) due to the double exchange. The energy of one such droplet has the form

$$
\begin{aligned}
E & =-t\left(z-\frac{\pi^{2} a^{2}}{R^{2}}\right)-\frac{W^{2}}{6 V z}\left[1-\frac{4}{3} \pi\left(\frac{R}{a}\right)^{3}\right]+ \\
& +z J S^{2} \frac{4}{3} \pi\left(\frac{R}{a}\right)^{3}-z J S^{2}\left[1-\frac{4}{3} \pi\left(\frac{R}{a}\right)^{3}\right] .
\end{aligned}
$$

The last two terms in (25) describe respectively the loss in the energy of the Heisenberg AFM exchange inside the FM metallic droplets and the gain of this energy in the AFM insulating part of the sample. The minimization with respect to the droplet radius (as in (21)) yields

$$
\frac{R}{a} \propto\left(\frac{t}{V}+\frac{J S^{2}}{t}\right)^{-1 / 5}
$$

Note that at $t / V \ll J S^{2} / t$, formula (26) gives just the same estimate for the radius of FM metallic droplet $(R / a) \sim\left(t / J S^{2}\right)^{1 / 5}$ as in 8, 14.

In the opposite limit when $(t / V) \gg J S^{2} / t$, we reproduce the same result $(R / a) \sim(V / t)^{1 / 5}$ as in (22). Finally, critical concentration $n_{c}$ is estimated as follows

$$
n_{c} \propto\left(\frac{t}{V}+\frac{J S^{2}}{t}\right)^{3 / 5}
$$

As a result, taking into account also the tendency to the phase separation at very small values of $n$ [8 11, 14] we come to the following phase diagram for the extended model (cf. [11]:

1. At $0<n<\left(\frac{J S^{2}}{t}\right)^{3 / 5}$, it corresponds to the phase separation into a FM metal with $n=n^{\prime}>0$ embedded in the AFM insulating matrix $(n=0)$. To minimize the Coulomb energy, it may be again favorable to split this metallic region into droplets with the concentration $n^{\prime}$ and an average radius given by Eq. (26) with $t / V=0$, each containing one electron and kept apart from one another.

2. At $\left(\frac{J S^{2}}{t}\right)^{3 / 5}<n<\left(\frac{t}{V}+\frac{J S^{2}}{t}\right)^{3 / 5}<1 / 2$, the system is a FM metal. Of course, we need a window of parameters to satisfy the inequality in the right-hand side. In actual manganites where $t / V \sim 1 / 2 \div 1 / 3$ and $J / t \sim 1 / 3$, these conditions upon $n$ are not necessarily satisfied. Experiments suggest that this window is present for $\mathrm{La}_{1-x} \mathrm{Ca}_{x} \mathrm{MnO}_{3}$, but it is definitely absent for $\operatorname{Pr}_{1-x} \mathrm{Ca}_{x} \mathrm{MnO}_{3}$ [11];

3. Finally, at $\left(\frac{t}{V}+\frac{J S^{2}}{t}\right)^{3 / 5}<n<\frac{1}{2}$, we have the phase separation in the form of FM metallic droplets inside the AFM charge ordered matrix.

This phase diagram is in a good qualitative agreement with many available experimental results for real manganites [22] - 25], in particular with the observation of the small-scale phase separation close to 0.5 doping [26]. Note also that our phase diagram has certain similarities with the phase diagram obtained in [28]29] for the problem of spontaneous ferromagnetism in doped excitonic insulators. 


\section{CONCLUSION}

Summarizing, we have shown that the narrow-band system, which has the checkerboard charge ordering at $n=1 / 2$ (corresponding to the doping $x=0.5)$ is unstable toward phase separation away from half-filling $(n \neq 1 / 2)$. It separates into the regions with the ideal CO $(n=1 / 2)$ and the other regions, in which extra electrons or holes are trapped. The simplest form of these metallic regions could be spherical metallic droplets embedded into the CO insulating matrix. Simple considerations allow us to estimate the size of these droplets and the critical concentration, or doping $x_{c}=1 / 2-\delta_{c}$, at which the metallic phase would occupy the whole sample and the CO phase would disappear. The account of the magnetic interactions does not change these conclusions but somewhat modifies the characteristic parameters of the metallic droplets.

The long-range Coulomb interaction may also modify the results, but we do not expect any qualitative changes. For the realistic values of parameters, the size of metallic droplets is still microscopic (about 10-30 ), and the excess charge in them will be rather small.

The obtained picture corresponds rather well to the known properties of 3D and layered manganites close to (less than) half doping, $x \leq 1 / 2$. Percolation picture of transport properties emerging from this treatment is confirmed by the results reported in [7,15,22,24,26]; moreover the coexistence of ferromagnetic reflections and those of the $\mathrm{CE}$ type magnetic structure typical of the CO state at $x=0.5$ were observed by the neutron scattering [27]. Thus, the general behavior of underdoped manganites $(x \leq 0.5)$ is in a good qualitative agreement with our results.

Our treatment leads to the same tendency to phase separation (instability of the homogeneous CO phase) also for overdoped regime, $x>0.5$. What would be the second phase in this case, is not yet clear. Therefore we did not concentrate our attention on such a situation.

Our treatment is applicable also to other systems with the charge ordering, such as cobaltites 18 and nickelates [19]. It would be interesting to study them for charge carrier concentrations different from the commensurate "checkerboard" one

A number of important problems still remain unsolved (the origin of the "in-phase" ordering in perovskite manganites in $c$-direction, the detailed description of inhomogeneous states in overdoped regime $n>1 / 2$, the behavior at

finite temperatures). Nevertheless, in spite of the introduced simplifications, our model seems to capture the essential physics underlying the interplay between phase separation and charge ordering in transition metal oxides.

\section{ACKNOWLEDGMENTS}

We are grateful to N.M. Plakida and M.S. Mar'enko for stimulating discussions. D.Kh. expresses gratitude to S.W. Cheong and Y. Moritomo for the discussions of the experimental aspects of the problem. The work was supported by INTAS (grants 97-0963 and 97-11954) and by the Russian-Dutch Program for Scientific Cooperation funded by the Netherlands Organization for Scientific Research (NWO). M.Yu.K. acknowledges the support of the Russian President Program (grant 96-15-9694). The work of D.Kh. was also supported by the Netherlands Foundation for the Fundamental Research of Matter (FOM) and by the European network OXSEN.

[1] E. Verwey, Nature (London) 144327 (1939); E. Verwey and P.W. Haayman, Physica 8, 979 (1941).

[2] D.I. Khomskii, Preprint of the P.N. Lebedev Physics Institute no. 105 (1969).

[3] T. Mutou and H. Kontani, Phys. Rev. Lett. 83, 3685 (1999).

[4] J. van den Brink, G. Khaliullin, and D. Khomskii, Phys. Rev. Lett. 83, 5118 (1999).

[5] G. Jackeli, N.B. Perkins, and N.M. Plakida, cond-mat/9910391; Phys. Rev. B (in press).

[6] Z. Jirak et al. J. Magn. Magn. Mater. 53, 153 (1985).

[7] A. Arulraj et al., Phys. Rev. B 56 R8115.(1998); M. Uehara et al. Nature 399, 560 (1999).

[8] E.L. Nagaev, Usp. Fiz. Nauk 166, 833 (1996) [Phys. - Uspekhi 39, 781 (1996)].

[9] A. Moreo, S. Yunoki, and E. Dagotto, Science 283, 2034 (1999).

[10] D. Arovas and F. Guinea, Phys. Rev. B 58, 9150 (1998).

[11] D.I. Khomskii, Physica B 280, 325 (2000).

[12] G.S. Uhrig and R. Vlamink, Phys. Rev. Lett. 71, 271 (1993). 
[13] S. Mori, C.H. Chen, and S.-W. Cheong, Nature (London) 392, 473 (1998).

[14] M.Yu. Kagan, D.I. Khomskii, and M.V. Mostovoy, Eur. Phys. J. B 12, 217 (1999).

[15] L.P. Gor'kov and V.Z. Kresin, JETP Letters 67, 985 (1998).

[16] S. Yunoki, T. Hotta, and E. Dagotto, Phys. Rev. Lett. 84, 3714 (2000).

[17] I.V. Soloviev and K. Terakura, Phys. Rev. Lett. 83, 2825 (1999).

[18] Y. Moritomo, M. Takeo, X.J. Liu, T. Akimoto, and A.Nakamura, Phys. Rev. B 58, R13334 (1998).

[19] J.A. Alonso, J.L. García-Muñoz, M.T. Fernández-Díaz, M.A.G. Aranda, M.J. Martínez-Lope, and M.T. Casais, Phys. Rev. Lett. 82, 3871 (1999).

[20] P. Pietig, R. Bulla, and S. Blawid, Phys. Rev. Lett. 82, 4046 (1999).

[21] P.B. Visscher, Phys. Rev. B 10, 943 (1974).

[22] N.A. Babushkina et al., J. Phys.: Condens. Matter 11, 5865 (1999).

[23] M. Hennion et al., Phys. Rev. Lett. 81, 1957(1998).

[24] G. Allodi, R. De Renzi, G. Guidi et al., Phys. Rev. B 56, 6036 (1997).

[25] I.F. Voloshin et al., Pis'ma v ZhETF 71, 157 (2000) [JETP Letters 71, 106 (2000)].

[26] Y. Moritomo, A. Machidas, S. Mori, N. Yamamoto, and A.Nakamura, Phys. Rev. B 60, 9220 (1999).

[27] R. Kayumoto, H. Yoshizawa, H. Kawano, Y. Tokura, K. Ohoyama, and M. Ohashi, Phys. Rev. B 60, 9506 (1999).

[28] L. Balents and C.M. Varma, Phys. Rev. Lett. 84, 1264 (2000).

[29] V. Barzykin and L.P. Gor'kov, cond-mat/9906401. 